\title{
Performance Evaluation and Comparison of Sahi Pro and Selenium Webdriver
}

\author{
Jagdish Singh \\ Student, UIET, Panjab University, \\ Chandigarh, India
}

\author{
Monika Sharma \\ Assistant Professor, UIET, Panjab University, \\ Chandigarh, India
}

\begin{abstract}
Software testing is a crucial phase in software development life cycle that reduce errors, cut maintenance and overall software costs. Manual testing takes a lot of time and effort, so automation tools are used by testers. There are number of automation testing tools available, but to choose the appropriate tool for the task requires various parameters to be considered. In this paper an evaluation on web-based software testing tools; Sahi Pro and Selenium Webdriver, based on execution time, recording and playback efficiency, browser and platform compatibility, result reporting, ease of learning and cost has been presented. It will help testers to take informed decision to choose a tool according to their requirements and resources.
\end{abstract}

\section{Keywords}

Automaiton testing tools, Sahi Pro, Selenium Webdriver.

\section{INTRODUCTION}

Software testing is a mechanism of assessment of the software which is intended to detect errors in it. The purpose of software testing is to evaluate the capability of the software and check that is of desired quality. The objective of software testing is to find out the faults present in the software. In software testing phase, the product is evaluated by a tester manually or using an automation tool to check that it meets the requirements or to check the disparity between expected and actual results.

Two types of testing are there. Manual testing; which is carried out by the testers physically. Tester plays the role of an end user and tries to explore the software to find out defects in it and to check the proper functioning of software. A test plan is written and followed. It is laborious and takes a lot of time and effort and there is a possibility that some errors may remain undiscovered. Automation testing is done by using an automation testing tool. It saves time and efforts, find hidden defects in the system.

Web-based applications are very common these days, because of the increasing use of internet. Various web automation testing tools are available to test performance, functional, security, load and stress testing, service testing of web applications. Tester must choose appropriate tools according to its requirement and its resources, otherwise testing will suffer, which lead to poor quality software. Because of a number of tools available these days, choosing the best tool for your task is tricky. Tester needs to check various parameters like execution speed, efficiency of recording and playback, result reporting, browser and platform compatibility, ease of learning and cost of various automation testing tools before selecting a tool. In this research a comparative evaluation of two web application testing tools: Sahi Pro and Selenium Webdriver have been done.
This paper is organized into five sections. Section I is the prelude of this paper, giving introduction about the topic and describing difference between automation testing and manual testing. Section II describes literature survey. In section III web automation tools used for evaluation is discussed and section IV discusses the testing environment. In section V results are discussed and finally the last section, section VI concludes the paper.

\section{LITERATURE REVIEW}

In paper [4], authors have described the basics about testing and three types of testing. This paper also gives the introduction to web testing and various web automation testing tools. Introduction to the top 10 web automation testing tools is given and some information like release date, language used, platform supported, browser supported etc. of each tool is given.

In paper [5], an approach has presented to mine executable specifications from Selenium IDE test suite. They evaluate a number of test suits for real world system. In their work they have demonstrated integration of two opposite processed of model mining and model based test integration in one framework.

In paper [6], authors have conducted a comparative study of automated tools, Mercury QuickTest Professional and QA TestComplete. The study is based on criteria such as the efforts involved in generating test scripts, result reports, playback capabilities, speed and cost. They have analyzed the features of above mentioned testing tools. The features help in minimizing the resources in script maintenance and increasing efficiency for script reuse. The authors have calculated each parameter and have provided the conclusion TestComplete has efficient playback and easy to use UI. QTP is best where data security is necessary.

In paper [7], authors have introduced the selenium framework. First the basics of selenium framework have discussed, and then the selenium suite is explained. Selenium suite has four tools Selenium IDE, Selenium RC, Selenium Webdriver, Selenium Grid. All tools are discussed thoroughly, showing the benefits and limitation of each tool.

In paper [8], authors have presented a comparative study of automated tools Selenium IDE, HP Quick test professional (QTP) and TestComplete (TC). They have evaluated and compared the three automated software testing tools on the basis of parameters such as recording efficiency, data driven testing, test result reports, reusability, capability of generation of scripts, playback of the scripts, execution speed, easy to learn and cost. In the conclusion they concluded QTP as the best tool amongst the three. 
In paper [9], authors have evaluated selenium suite to find the best tool in the suite and then compare the best tool in the selenium suite with the Watir tool. Out of Selenium suite, Selenium WebDriver is the best tools although selenium IDE is rated higher according to performance evaluation but it is not taken because it is only a plug in for Firefox. Also selenium RC is not recommended because it uses Selenium $\mathrm{RC}$ sever to run selenium RC test and as for selenium grid it is used for parallel test it is also not recommended. Then they performed the evaluation of Selenium WebDriver and Watir Webdriver by taking parameters like Execution speed, Record and Playback capability, Browser Compatibility, Platform Compatibility, Programming Language Supported, Test Result Report and Future in terms of Usability and Accessibility. In their results they concluded that Selenium WebDriver is better than Watir WebDriver.

In paper [10], authors have presented comparatively analysis of Sahi Open Source and Selenium IDE based on parameters such as execution speed, installation and configuration, record and playback, logging and reporting, cost and platform compatibility. Towards the end authors have concluded that Selenium IDE is aimed at and best be used by programmers and Sahi is aimed at non-programmer testers. Selenium IDE works for only Mozilla Firefox whereas Sahi works for all browsers.

\section{WEB AUOMATION TESTING TOOLS}

A number of web-based automation testing tools are available in the market. Using automation testing tools test coverage increases that reduce the time required to test the software and ultimately reduces manual efforts. To choose the appropriate tool for the task, the tester is required to evaluate the certain parameters of the tools. The tools chosen for this research have been described below.

\subsection{Sahi Pro}

Sahi Pro is a commercial web automation testing tool. It is also available as OS (Open Source), free product with some basic features. Sahi Pro is a cross-browser and cross platform testing tool. It works well in agile development environment, thus enabling swift automation, maintenance and easy integration with build systems. It saves time and effort with faster development, less maintenance and fast distributed playback. Sahi runs on any modern browser which supports JavaScript. Sahi Pro is chosen because of features provided in it that are missing in Sahi open source.

\subsection{Selenium Webdriver}

Selenium Webdriver is one of the four tools in Selenium suite. It is a cross-browser and cross-platform testing tool. It is an open source tool that can be easily downloaded from Selenium website. It supports different programming languages.

\section{TESTING ENVIRONMENT}

The test environment consists of logical setup that includes two types of open source web automation tools called Sahi Pro and Selenium Webdriver. Specification of system on which test is done is shown below:

Processor: Intel® Core ${ }^{\mathrm{TM}} 2$ Duo $2.20 \mathrm{GHz}$ CPU

RAM: $3.00 \mathrm{~GB}$

System Type: 32-bit Operating System

OS: Windows 7 Professional
Evaluation between Sahi Pro and Selenium Webdriver was done on Mozilla Firefox and Google Chrome and test cases are written to test the login function of four web applications namely Gmail, Yahoo Mail, Facebook and Twitter. To increase the efficiency and quality during the course of executing the tests with each particular tool, the background services of other programs as well as any other application were either terminated.

\section{RESULTS}

This section compares the Sahi Pro and Selenium Webdriver and presents the graphs of the evaluation of these tools. Sahi Pro and Selenium Webdriver are evaluated and compared based on parameters described below:

\subsection{Execution Time}

The following bar graphs show the execution time of each test case in Mozilla Firefox and Google Chrome.

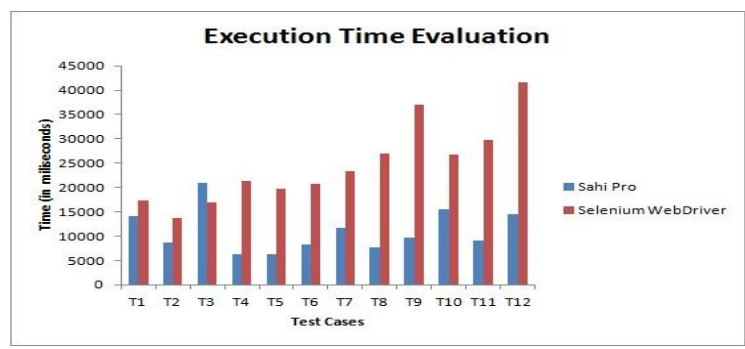

Fig.1. Execution time Evaluation of Sahi Pro and Selenium Webdriver in Mozilla Firefox

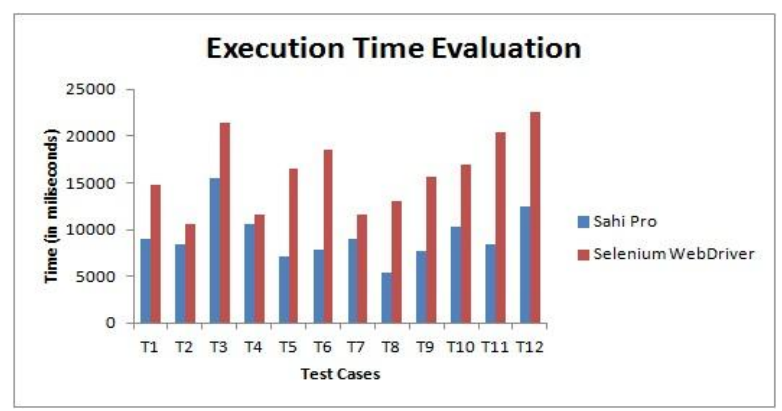

Fig.2. Execution time Evaluation of Sahi Pro and Selenium Webdriver in Google Chrome

From the above figure it is clear that Sahi Pro perform better than Selenium Webdriver in terms of execution speed. Average execution to execute a test in Sahi Pro is $11071 \mathrm{~ms}$ for Mozilla Firefox and $9301 \mathrm{~ms}$ for Google Chrome and for Selenium Webdirver it is $24606 \mathrm{~ms}$ in case of Mozilla Firefox and $16126 \mathrm{~ms}$ for Google Chrome. It is also clear that both tools perform better in Google Chrome than in Mozilla Firefox.

\subsection{Recording Efficiency}

Sahi Pro is an excellent recorder and it spy on objects which works on all modern browsers. In Sahi Pro tester can add assertion, see automated documentation about recorded steps where as Selenium Webdriver does not support recording tool. So for non programmer Sahi Pro is the better option.

\subsection{Playback Capabilities}

Sahi Pro is a good playbook tool. It has pause option to pause the execution of a script, shows the step by step execution of script whereas Selenium Webdriver lacks in playback capabilities. Tester cannot pause the execution of test once it 
has started, tester can only stop it. Step by step execution is not shown.

\subsection{Browser Compatibility}

Both Sahi Pro and Selenium Webdriver are compatible with all modern web browsers. In Sahi Pro any browser can be added by doing some simple configuration steps. With Selenium Webdriver testers can work with any browser by invoking appropriate packages and writing code respective to the browser.

\subsection{Platform Compatibility}

Sahi Pro works on Windows and Linux but Selenium Webdriver is a cross platform testing tool.

\subsection{Programming language supported:}

Sahi Pro supports Java, JavaScript and Sahi script. Selenium Webdriver supports domain specific languages which are Java, C\#, Ruby and Python. Selenium provides more option for tester while choosing programming languages.

\subsection{Test Result Report}

Sahi Pro supports a good result reporting feature. The reports are simple to understand and tester can make analysis and locate the problem easily. Sahi Pro has a result log, where all the results are stored. Tester can export results in Excel format in Sahi Pro. Selenium Webdriver does have any result reporting feature.

\subsection{Ease of learning}

Sahi Pro is recording and playback tool with simple steps for dealing with the tool. A non-programmer can work with this tool. Therefore it is easy to do testing with Sahi Pro. Selenium Webdriver takes a lot of time in its installation and configuration. For Selenium Webdriver tester needs to have programming skills. It takes time to work with it.

\subsection{Cost}

Sahi Pro is not an open source tool. It costs around Rs 32,000 per user for a year in India and $695 \$$ outside India. It is quite expensive, which can be a constraint for its selection. Selenium Webdriver is an open source tool. So a tester has to pay nothing for it.

For the purpose of rating of the tools, 5- point rating criteria i.e. 1 to 5 is used. Rating 5 presents extremely good remarks for the tool whereas rating 1 presents very poor remarks for the tool. On the basis of above parameters an evaluation bar graph is made shown below, which gives evaluation detail.

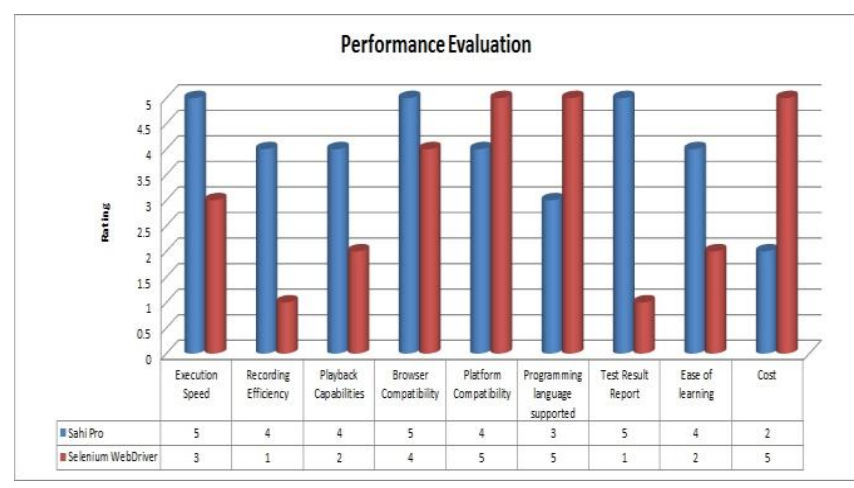

Fig.3. Performance Evaluation of Sahi Pro and Selenium Webdriver

\section{CONCLUSION}

Automated software testing tools has become necessity of software companies because it saves both time and money in the testing phase. In this paper evaluation of two web-based automation testing tools; Sahi Pro and Selenium Webdriver has been presented. Evaluation is done on the basis of parameters, which are crucial in software testing. From the evaluation it is clear that Sahi Pro performs better than Selenium Webdriver in case of execution time. Both tools are browser independent but both perform faster in Mozilla Firefox than in Google Chrome. According to the recording and playback criteria, Sahi Pro is better than Selenium Webdriver. Selenium is a better choice in case of platform compatibility and programming language support. Sahi Pro has very good result reporting feature, which is missing in Selenium Webdriver. Sahi Pro is easy to learn, even a nonprogrammer can work with it but Selenium requires some programming skills. Selenium Webdriver is open source, so free of cost but Sahi Pro is costly. It is difficult to choose the best tool for the task, from the above evaluation it is easy for tester to choose between Sahi Pro and Selenium Webdriver according to his requirements and system specifications.

Towards future, performance evaluation of various web applications can be done for more browsers. Another future directive can be to test the web applications on the above mentioned web automation testing tools on different platforms. The testing of web applications in other languages (like .net, php, python etc.) can also be considered in future.

\section{REFERENCES}

[1] A. Gargantini and E. Riccobene, "ASM-Based Testing: Coverage Criteria and Automatic Test Sequence," J.UCS: Journal of Universal Computer Science, pp. 1050-1067, 2001.

[2] F. Wang, W. Du, "A Test Automation Framework Based on WEB," in Proceedings of the $11^{\text {th }}$ International Conference on Computer and Information Science. IEEE/ACIS, pp. 683-687, 2012.

[3] B. Haugset, G. K. Hanssen, “Automated Acceptance Testing: a Literature Review and an Industrial Case Study", in Proceedings of the Agile 2008 Conference, IEEE Computer Society, pp. 27-32, 2008.

[4] Monika Sharma, Rigzin Angmo,"Web based Automation testing and Tools", , International Journal of Computer Science and Information Technonologies (IJCSIT), ISSN: 0975-9646, Vol. , ISSUE 1, pp. 908-912, 2014.

[5] Bharath Cheluvaraju, Kartikay Nagal, Anjaneyulu Pasala,"Mining Software Revision History using Advanced Social Network Analysis", 19th Asia-Pacific Software Engineering Conference, pages 717-720, IEEE, 2012.

[6] Manjeet. Kaur, Raj. Kumari, "Comparative Study of Automated Testing Tools: TestComplete and QuickTest Pro", International Journal of Computer Application, vol 24, no. 1, 2011.

[7] Rigzin Angmo, Monika Sharma, "Selenium Tool: A Web based Automation Testing Framework", IJETCAS, 2279-0055.

[8] H. Kaur,Dr. G. Gupta, "Comparative Study of automation testing tools:selenium, quick test professional and testcomplete," International Journal of Engineering 
Research and Application, vol. 3, no. 5, pp. 1739-1743, 2013.

[9] Rigzin Angmo, Monika Sharma, "Performance Evaluation of Web Based Automation Testing Tools", $5^{\text {th }}$ International Conference- Confluence The Next Generation Information Technology Summit, pp. 731$735,2014$.

[10] Thanuja Naidu, Nor Basri, Saravanan Nagenthram, "SAHI vs. Selenium: A Comparative Analysis" IEEE, pp. 967-970, 2014

[11] R. Rattan and Shallu, "Performance Evaluation \& Comparison of Software Testing Tool," International Journal of Information and Computation Technology, vol 3, no. 7, pp. 711-716, 2013.

[12] M. Sharma, R. Angmo,"Web based Automation testing and Tools," International Journal of Computer Science and Information Technonologies (IJCSIT), vol. 5, no. 1, pp. 08-912, 2014.

[13] I. Singh, B. Tarika, "Comparative Analysis of Open Source Automated Software Testing Tools: Selenium, Sikuli and Watir" International Journal of Information \& Computation Technology, vol 4, pp. 1507-1518, 2015.
[14] Rafi, "Benefits and limitations of automated software testing: Systematic literature review and practitioner survey", Automation of Software Test, IEEE, pp. 36-42, 2012.

[15] http://en.wikipedia.org/wiki/Test_automation

[16] http:/www.seleniumhq.org

[17] B. Kitchenham and S. Charters, "Guidelines for Performing Systematic Literature Reviews in Software Engineering," Software Engineering Group, School of Computer Science and Mathematics, Keele University, Tech. Rep. EBSE-2007-01, July 2007.

[18] N. Nie, "Reliability and Performance Testing Model of Web_Based User Login and Access Control", IEEE, pp $1-4,2010$.

[19] S. Shoney, "An Adaptive Framework for Web Services Testing Automation Using JMeter", IEEE, pp. 314-418, 2014.

[20] Z. Wandon, "Design and Implementation of a Web Application Automation Testing Framework", IEEE, pp. 316-318, 2009. 